\title{
Complex film of chitosan and carboxymethyl cellulose nanofibers
}

Takuma Kawasaki ${ }^{a}$, Tadashi Nakaji-Hirabayashi ${ }^{\text {a,b }}$, Kazuhira Masuyama ${ }^{\text {a }}$, Satoshi Fujita ${ }^{c}$, Hiromi $\operatorname{Kitano}^{\mathrm{a}^{*}}$

${ }^{\text {a }}$ Department of Applied Chemistry, Graduate School of Science and Engineering, University of Toyama, 3190 Gofuku, Toyama-shi, Toyama 930-8555, Japan

${ }^{\mathrm{b}}$ Frontier Research Core for Life Sciences, University of Toyama, 3190 Gofuku, Toyama-shi, Toyama 930-8555, Japan

${ }^{\mathrm{c}}$ Department of Fiber Technology and Sciences, Graduate School of Engineering, University of Fukui, 3-9-1 Bunkyo, Fukui-shi, Fukui 910-8507, Japan

\section{*Corresponding author: Hiromi Kitano}

E-mail address: kitano@eng.u-toyama.ac.jp

Number of Words: Abstract, 131. Text (from Introduction to Conclusions), 1980.

Number of Figures, 5.

Number of Tables, 2.

Number of Scheme, 1. 
Abstract: A polymer film composed of a mixture of chitosan $(\mathrm{Ch})$ and carboxymethyl cellulose sodium salt (CMC) nanofibers was deposited on a glass surface. The thin film of the Ch-CMC mixture obtained was stable, and fibroblast adhesion to the film was lowest when the weight ratio of Ch to $\mathrm{CMC}$ was 4:6. The $\zeta$-potential and contact angle of the mixture film indicated that a polyion complex of $\mathrm{Ch}$ and $\mathrm{CMC}$ was formed. The mechanical strength of the film composed of Ch-CMC nanofiber complexes was much higher than that of the film composed of Ch-water-soluble CMC complexes (non-nanofiber), likely because the entanglement of nanofibers was enhanced by electrostatic attractions. These results indicate that the charge-neutralized nanofiber film was highly effective in suppressing cell adhesion and therefore is a promising material for biomedical applications. 131 Words

Keywords: anti-biofouling, carboxymethyl cellulose, chitosan, nanofiber film, polyion complex

\section{Introduction}

Zwitterionic polymers have been designed to mimic phosphatidylcholine (lecithin), which is abundant in cell membranes [1,2], and their applicability in biomedical fields has extensively been investigated. For example, polymer films composed of apolar monomers such as $n$-butyl methacrylate (BMA) and zwitterionic monomers such as 2-methacryloyloxyethyl phosphorylcholine (phosphobetaine), 3-sulfo- $N, N$-dimethyl- $N$-(3-methacrylamidopropyl)propanaminium inner salt (sulfopropylbetaine), and 1-carboxy- $N, N$-dimethyl- $N$-(2-methacryloyloxyethyl)methanaminium inner salt (CMB, carboxymethylbetaine) were found to be highly biocompatible [3-16]. We previously reported that the amount of proteins adsorbed and the number of platelets adhered onto a film of a random copolymer of CMB and BMA were much lower than that on a polyBMA film $[8,9]$.

Similarly, the charge-neutralized surface was found to be bio-inert. A self-assembled monolayer 
of a 1:1 mixture of positively and negatively charged alkyl mercaptans, for example, was found to be resistant to protein adsorption [17]. A terpolymer film composed of $76 \mathrm{~mol} \%$ of BMA and equal amounts of negatively and positively charged monomer residues (methacrylic acid (MA) and 3-(dimethylamino)propyl methacrylamide (DMAPMA), respectively) was also found to be bio-inert $[18]$.

Polyion complexes (PICs) have been extensively used in many bio-related applications and research fields, including as cell film carriers using PIC gel-modified membranes [19], bio-based food packaging polymers [20], cellulose wet wiper films [21], composite membranes for dehydration of ethanol/water azeotrope [22], ultralong polyion nanoladders facilitated by ionic recognition [23], ocular drug delivery for bioactive proteins [24], and preparation of submicrometer-sized unilamellar PIC vesicles for drug delivery systems [25].

Recently, nanofibers from biomass have been gaining attention because the nanofibers can be easily prepared from various types of biomass. For example, cellulose nanofibers consisting of kraft pulp have been applied to transparent paper (1) by polishing using emory paper (without any additives), (2) by the lamination of transparent plastics (polycarbonate films), and (3) by the deposition of transparent acrylic resins [26]. Acrylic resin nanocomposites combined with chitin nanofibers obtained from crab shells as mechanical-reinforcing agents are promising candidates as substrates for the manufacturing of optoelectronic devices such as bendable displays, flat panel displays, and solar cells [27].

The fibrillation of pulp fiber has mainly been performed by two methods, by using either a grinder or a high-pressure homogenizer. The former method results in the successful fibrillation of wood pulp fibers into nanofibers, and provides films with better transparency than that obtained by the latter method after the deposition of acrylic resins [28]. Recently, another fibrillation method has been reported; a water-jet method [29], which requires neither chemicals nor heat energy.

Composite materials composed of chitosan $(\mathrm{Ch})$ or carboxymethyl cellulose nanofibers and other polymers such as alginate and poly(vinyl alcohol) have been developed and examined for 
biomaterial applications, such as in artificial extracellular matrices and wound dressing [30-32]. However, functional biomaterials using only chitosan and carboxymethyl cellulose nanofibers may be developed based on their advantageous mechanical and electrostatic properties. In this study, a thin film of PIC, composed of a mixture of $\mathrm{Ch}$ and carboxymethyl cellulose sodium salt (CMC) nanofibers, was prepared on the surface of a glass substrate by using the cast method, and the adhesion behavior of cells to the film was investigated. Charge neutralization was found to be critical for biocompatibility of the surface of polymer, metal and ceramic materials.

\section{Material and Methods}

\section{Materials}

CMC and Ch nanofiber suspension (2\% in water), which had been prepared by using a unique atomizing system (Star Burst System) [29], were donated by Sugino Machine Ltd. (Uozu, Toyama, Japan). Water-soluble CMC and Chitosan 10 were purchased from Nacalai Tesque Inc. (Kyoto, Japan) and Wako Pure Chemical Industries Ltd. (Osaka, Japan), respectively. Other reagents used were commercially available. All aqueous solutions were prepared in ultrapure water $(18 \mathrm{M} \Omega . \mathrm{cm}$, EMD Millipore, Billerica, MA, USA).

\section{Construction and characterization of PIC films}

A slide glass (Matsunami Glass Ind., Ltd., Osaka, Japan) was cut into a $24 \times 26 \mathrm{~mm}$ tip and pretreated with an ozone cleaner at room temperature for $1 \mathrm{~h}$. In order to prepare the square PIC film, a silicon frame (thickness, $1.0 \mathrm{~mm}$; square window, $21 \times 23 \mathrm{~mm}$; incubated in $\mathrm{MeOH}$ for $24 \mathrm{~h}$ to remove plasticizer prior to use) was placed on the glass, and a $0.5 \mathrm{wt} \%$ suspension of the nanofiber mixture $(600 \mu \mathrm{L})$, which had been thoroughly dispersed in water by using a vortex mixer for $3 \mathrm{~min}$, was poured into the frame. The glass tip was dried over silica gel in a desiccator at room temperature for $48 \mathrm{~h}$ and the silicone frame was removed. At the preparation of non-nanofiber complex PIC film, carboxymethyl cellulose sodium salt and chitosan 10 dissolved in $0.05 \mathrm{M} \mathrm{HCl}$ 
were used, and the PIC films were prepared by the same method as that for nanofiber PIC film.

Contact angles, $\theta$, of pure water $(3-4 \mu \mathrm{L})$ at $30 \mathrm{~s}$ after deposition on the surface of polymer films constructed on glass substrates were measured at $23^{\circ} \mathrm{C}$ and $60 \%$ relative humidity by using the sessile drop method (CA-D, Kyowa Interface Science, Tokyo, Japan). The $\theta$ values were determined at 5 points, and the values were averaged as reported elsewhere [33,34]. All measurements were conducted in air at room temperature. The surface $\zeta$-potentials of the nanofiber complex films constructed on glass substrates $(35 \times 15 \mathrm{~mm})$ were measured in a $10 \mathrm{mM} \mathrm{NaCl}$ aqueous solution at $25{ }^{\circ} \mathrm{C}$ by using $\zeta$-potential analyzer (ELSZ-2, Otsuka Electronics Co. Ltd., Osaka, Japan). The thickness of the complex film was evaluated by laser scanning microscopy (Nanoscope IIIa, Bruker, Billerica, MA). Moreover, the degree of deacetylation of pure Ch and Ch nanofibers and the degree of carboxymethylation of pure CMC and CMC nanofibers were determined by conductometric analysis. Dried samples (Ch: $10 \mathrm{mg}$ and CMC: $30 \mathrm{mg}$ ) were uniformly dispersed in Milli-Q water $(10 \mathrm{~mL})$, and then $1.0 \mathrm{~mL}$ of $0.1 \mathrm{M} \mathrm{HCl}$ was added into the suspension. Fifty microliters of $0.1 \mathrm{M} \mathrm{NaOH}$ was added at appropriate intervals, and the conductivity of the suspension was measured at $25{ }^{\circ} \mathrm{C}$. The degree of deacetylation and carboxymethylation were determined from the inflection point of the obtained conductivity curve.

\section{Mechanical strength of non-fiber film}

The mechanical strength of various polymer films was evaluated by using a high-sensitive tensile testing machine (KES-G1-SH, Kato Tech Co., Ltd., Kyoto, Japan). The nanofiber film was prepared from a $1 \mathrm{w} / \mathrm{v} \% \mathrm{Ch}-\mathrm{CMC}$ mixture $(1200 \mu \mathrm{L})$. The film could be easily peeled from the silicon film attached to glass plate. A slip of the film 5-10 $\mathrm{mm}$ in width was suspended by using a clamp. The distance between the clamps was set to $5 \mathrm{~mm}$. The relationship between elongation and loading of these nanofiber or non-nanofiber films constructed with various contents of $\mathrm{Ch}$ and $\mathrm{CMC}$ were measured, and the maximum loading and stretch at fracture was determined. The tensile strength $\left(\mathrm{MPa}=\mathrm{N} / \mathrm{mm}^{2}\right)$ of each film was determined using the following equation. 
2 Tensile strength $(\mathrm{MPa})=\frac{\text { maximum load at break }(\mathrm{gf}) \times 9.81 \times 10^{-3}(\mathrm{~N} / \mathrm{gf})}{\text { sectional area }\left(\mathrm{mm}^{2}\right)}$

\section{Adhesion test of fibroblasts}

NIH 3 T3 cells were used for a cell adhesion assay. Single cells were obtained from culture at passage 141 by treatment with $0.25 \%$ trypsin- $1 \mathrm{mM}$ ethylenediaminetetraacetic acid solution. Cells suspended in culture medium (minimum ethential medium (MEM) containing 10\% fetal bovine serum, penicillin $(100 \mathrm{U} / \mathrm{mL})$, and streptomycin $(100 \mu \mathrm{g} / \mathrm{mL}))$ were seeded onto the PIC-modified substrate (sterilized with $70 \%$ ethanol aqueous solution) at a density of $5 \times 10^{4}$ cells $/ \mathrm{cm}^{2}$. The seeded cells were incubated for $12 \mathrm{~h}$ at $37^{\circ} \mathrm{C}$ and $5 \% \mathrm{CO}_{2}$ and washed with fresh culture medium to remove weakly adherent cells. The adhered cells were cultured for an additional $12 \mathrm{~h}$ at $37^{\circ} \mathrm{C}$ in $5 \%$ $\mathrm{CO}_{2}$; after incubation for $15 \mathrm{~min}$ with the culture medium containing $2 \mu \mathrm{g} / \mathrm{mL}$ Hoechst 33342 (Dojindo Laboratories, Kumamoto, Japan) and $1 \mu \mathrm{g} / \mathrm{mL}$ calcein-AM (Dojindo Laboratories), the cells were washed with PBS. Stained cells were observed by using an epifluorescence microscope (DP71, Olympus Optical Co., Ltd., Tokyo, Japan). Cells were counted on 5 different microphotographs obtained from the same sample and the data were averaged. The data are shown as the mean \pm standard deviation for 4 independent samples.

\section{Results and Discussion}

\section{Characterization of PIC films}

The degree of substitution for sodium $\mathrm{CMC}$ and the degree of deacetylation for $\mathrm{Ch}$ were determined by conductometric titration (Figure S1, Table 1). The degree of substitution for CMC nanofibers was much smaller than that for water-soluble CMC. This is because the solubility in water must be reduced to maintain the nanofiber structure. For $\mathrm{Ch}$, however, the degrees of deacetylation for nanofiber and non-nanofiber samples were similar and large, likely because of the inherent low solubility of Ch. 
Figure 1 shows that the contact angle of the nanofiber film varied with the contents of $\mathrm{CMC}$ and Ch. At a Ch content of 60-70 wt\% the nanofiber film showed the highest hydrophilicity, which is in accordance with the tendency for the non-nanofiber film composed of natural CMC and Ch. Moreover, the contact angle of the Ch nanofiber film was larger than that of the pure $\mathrm{Ch}$ film. This is probably because amine substituents of Ch might have been exposed to the outside environment during the preparation of nanofibers using the water-jet method, and partly because the nanofiber material had an inherent roughness. In fact, the contact angle of charge-neutralized $\mathrm{Ch} / \mathrm{CMC}$ nanofiber complex films was 25 - 30 degrees, whereas that of charge-neutralized and zwitterionic polymer brushes and films is $10-15$ degrees [35,36]. These results and previous data suggest that the different tendencies between nanofiber and non-nanofiber consisting of $100 \% \mathrm{Ch}$ and the increase of contact angle of nanofiber film were caused by increased roughness [37].

Figure 2 shows the $\zeta$-potential of the nanofiber film. The $\zeta$-potential increased with increasing Ch content. The data at 60 and $70 \mathrm{w} / \mathrm{v} \%$ of Ch slightly deviated from the linear relationship, likely because of the partial detachment of the film, resulting in the exposure of a glass substrate with a largely negative $\zeta$-potential $(\mathrm{ca} .-60 \mathrm{mV})[34,38]$. The non-nanofiber film was not stably attached to the glass substrate, making it impossible to measure the $\zeta$-potential.

The $\zeta$-potentials of nanofiber films at the $40-50 \%$ and $60-70 \%$ of $\mathrm{Ch}$ were slightly negative and almost zero, respectively. We thought that these results were due to a largely negative $\zeta$-potential of the glass substrate. The film might have been partially detached due to the use of 10 $\mathrm{mM} \mathrm{NaCl}$ aqueous solution during the zeta-potential measurement. The charge-neutralized films of $\mathrm{Ch}$ and $\mathrm{CMC}$ nanofiber could easily be detached from the glass surface by the incubation in phosphate buffer saline (saline concentration: $150 \mathrm{mM}$ ) at $37{ }^{\circ} \mathrm{C}$ for $10-20 \mathrm{~min}$. Therefore, the zeta-potentials of nanofiber films at $40-50 \%$ and $60-70 \%$ of Ch would be almost zero and slightly positive, respectively, if there was no influence of $\zeta$-potential of the glass substrate.

The charge-neutralized nanofiber complex of $\mathrm{Ch}$ and $\mathrm{CMC}$ was expected to have a smaller amount of $\mathrm{Ch}(\mathrm{Ch} \%=30-50)$ because the degree of deacetylation of $\mathrm{Ch}$ nanofiber was high (over 
$90 \%$ ) and the degree of carboxymethylation of CMC was low (under 30\%). Overall, the results obtained in the $\zeta$-potential measurements were reasonable.

The mechanical strength of nanofiber film was evaluated (Table 2, Figure 3 and S2). The thickness of nanofiber films prepared from $1200 \mu \mathrm{L}$ of $1 \%$ nanofiber mixture was $80-160 \mu \mathrm{m}$, whereas that of the non-nanofiber films prepared in the same manner was 170-290 $\mu \mathrm{m}$, likely because of the lower density of entangled water-soluble polymer chains compared to entangled nanofibers and the influence of the excess charge of counter-polymers. The non-nanofiber films of 50 and $60 \mathrm{w} / \mathrm{v} \% \mathrm{Ch}$ did not have sufficient strength for measurements, and those of $70 \mathrm{wt} \%$ and $100 \mathrm{wt} \%$ Ch did not give a film after drying.

The result showed that the nanofiber film was much tougher than the non-nanofiber film. The mixture of non-nanofiber $\mathrm{Ch}$ and CMC did not give a stable film at mixing ratios of 50:50-100:0. The micrograph of nanofiber showed the associated structure to form loose, three-dimensional networks [29]. Because of the entanglement of nanofibers, the mechanical strength of the nanofiber film may be greatly increased.

Moreover, the difference in tensile strength $(\sigma)$ relative to the composition ratio of $\mathrm{Ch}$ and CMC was investigated (Figure 3). Based on these results, the nanofiber film at $40 \%$ Ch content showed the highest $\sigma$ value. In contrast, the $\sigma$ values of nanofiber films containing $30 \%$ and $70 \% \mathrm{Ch}$ were much smaller than those of pure $\mathrm{Ch}$ or $\mathrm{CMC}$ nanofiber films, respectively. The highest tensile strength of the nanofiber film at a Ch content of $40 \%$ may have been derived from the strong electrostatic interaction resulting in the charge neutralization between $\mathrm{Ch}$ and $\mathrm{CMC}$. In contrast, the $\sigma$ of nanofiber films constructed with a Ch-CMC mixture at the high content of $\mathrm{Ch}$ or CMC (e.g. $30 \% \mathrm{Ch}$ and $70 \% \mathrm{Ch}$ ) may have been weak probably because of microphase separation associated with the formation of charge-neutralized domains besides positively- or negatively-charge domains.

\section{Cell adhesion}

Figure 4 shows the number of 3T3 cells adhered to the nanofiber film with various contents. The 
film with a Ch content of $40 \%$ was the least adhesive. Figure 5 shows fluorescence images of the cells attached to various nanofiber films. At a Ch content of $40 \%$, nearly complete suppression of cell adhesion was observed.

Previously, it was found that the number of platelets adhered to the surface of terpolymer (poly(BMA-MA-DMAPMA) was lowest when the contents of methacrylic acid (MA) and 3-(dimethylamino)propyl methacrylamide (DMAPMA) were comparable [18]. Similarly, the surface of the copolymer brush of MA and 2-(dimethylamino)ethyl methacrylate (DMAEMA) prepared at a feeding ratio of 1:1 was highly resistant to protein adsorption and cell adhesion compared with those adsorbed and adhered to the PMA and PDMAEMA brushes [38]. Therefore, taking into account the difference in the degree of nanofiber substitution (CMC 27.3\% and Ch $92.0 \%)$, the results in the figure can be explained as described above.

The correlation between inert properties of polymer materials to vicinal water and their biocompatibility has previously been assessed by considering the structure of water in the vicinity of charge-neutralized and zwitterionic polymers, which was investigated using Raman, infrared, and sum frequency generation spectroscopies [39-44]. These studies suggest that the surface of amphoteric copolymers containing comparable content of MA and DMAEMA drastically suppresses both the orientation of vicinal water molecules, and the adsorption/adhesion of biomolecules such as proteins and cells [39]. The observed biocompatibility of the $\mathrm{Ch} / \mathrm{CMC}$ nanofiber complex examined in this study may be due to the same reason, strongly indicating that charge neutralization is heavily related to the biocompatibility of the nanofiber films.

\section{Conclusions}

The mixed film of $\mathrm{Ch}$ and CMC was resistant to non-specific adsorption of fibroblasts because of charge neutralization at the surface. Because of entanglement supported by electrostatic attractions, the complex nanofiber film was sufficiently tough to protect a glass substrate from non-specific cell adhesion. Consequently, it was definitely suggested that the materials composed of $\mathrm{Ch}$ and CMC nanofiber complex is hopeful as functional biomaterials. 


\section{Acknowledgements}

This research was supported by a Grant-in-Aid for Scientific Research (26188100, 15H05353) from the Japan Society for the Promotion of Science (JSPS) and a Grant-in-Aid for Scientific Research on Innovative Areas (20106007) from the Ministry of Education, Culture, Sports, Science and Technology (MEXT), Japan. T. N.-H. is grateful to JST for the tenure-track program. We are indebted to Sugino Machine Ltd. for the donation of CMC and CT nanofibers.

\section{References}

[1] T. Nakaya and Y.-J. Li, Progr. Polym. Sci., 24 (1999) 143.

[2] A.L. Lewis, Colloids Surf. B: Biointerfaces, 18 (2000) 261.

[3] K. Ishihara, R. Aragaki, T. Ueda, A. Watanabe and N. Nakabayashi, J. Biomed. Mater. Res., $24(1990) 1069$.

[4] K. Ishihara, Sci. Technol. Adv. Mater., 1 (2000) 131.

[5] J. Yuan, C. Mao, J. Zhou, J. Shen, S.C. Lin, W. Zhu and J.F. Fang, Polym. Int., 52 (2003) 1869.

[6] Y. Yuan, X. Zang, F. Ai, J. Zhou, J. Shen and S. Lin, Polym. Int., 53 (2004) 121.

[7] H. Kitano, T. Mori, S. Tada, Y. Takeuchi, M. Gemmei-Ide and M. Tanaka, Macromol. Biosci., $5(2005) 314$.

[8] H. Kitano, S. Tada, T. Mori, K. Takaha, M. Gemmei-Ide, M. Tanaka, M. Fukuda and Y. Yokoyama, Langmuir, 21 (2005) 11932.

[9] S. Tada, C. Inaba, K. Mizukami, S. Fujishita, M. Gemmei-Ide, H. Kitano, M. Tanaka, A. Mochizuki and T. Matsunaga, Macromol. Biosci., 9 (2009) 63.

[10] S. Fujishita, S. Tada, C. Inaba, M. Gemmei-Ide, H. Kitano and Y. Saruwatari, Biol. Pharm. Bull., 31(2008) 2309.

[11] E.J. Lobb, I.Y. Ma, N.C. Billingham, S.P. Armes and A.L. Lewis, J. Am. Chem. Soc., 123 (2001) 7913. 
[12] I.Y. Ma, E.J. Lobb, N.C. Billingham, S.P. Armes, A.L. Lewis, A.W. Lloyd and J. Salvage, Macromolecules, 35 (2002) 9306.

[13] I.Y. Ma, Y. Tang, N.C. Billingham, S.P. Armes, A.L. Lewis, A.W. Lloyd and J.P. Salvage, Macromolecules, 36 (2003) 3475.

[14] Q.S. Mu, J.R. Lu, Y.H. Ma, H.V. Paz de Banez, K.L. Robinson, S.P. Armes, A.L. Lewis and R.K. Thomas, Langmuir, 22 (2006) 6153.

[15] Y. Li, Y. Tang, R. Narain, A.L. Lewis and S.P. Armes, Langmuir, 21 (2005) 9946.

[16] Y. Li, S.P. Armes, X. Jin and S. Zhu, Macromolecules, 36 (2003) 8268.

[17] R.E. Holmlin, X. Chen, R.G. Chapman, S. Takayama and G.M. Whitesides, Langmuir, 17 (2001) 2841.

[18] H. Kitano, K. Nagaoka, S. Tada, M. Gemmei-Ide and M. Tanaka, Macromol. Biosci., 8 (2008) 77.

[19] Z. Tang, A. Kikuchi, Y. Akiyama and T. Okano, React. Func. Polym., 67 (2007) 1388.

[20] S. Farris, K.M. Schaich, L.S. Liu, L. Piergiovannia and K.L. Yam, Trends Food Sci. Technol., 20 (2009) 316.

[21] T. Uematsu, Y. Matsui, S. Kakiuchi and A. Isogai, Cellulose, 18 (2011) 1129.

[22] T. Uragami, S. Yamamoto and T. Miyata, Biomacromolecules, 4 (2003) 137.

[23] L. Xu, L. Jiang, M. Drechsler, Y. Sun, Z. Liu, J. Huang, B.Z. Tang, Z. Li, M.A. Cohen Stuart, and Y. Tan, J. Am. Chem. Soc., 136 (2014) 1942.

[24] T. Yasulawa, Y. Tabata, H. Kimura and Y. Ogura, Expert Rev. Ophthalmol., 6 (2001) 657.

[25] Y. Anraku, A. Kishimura, M. Oba, Y. Yamasaki and K. Kataoka, J. Am. Chem. Soc., 132 (2010) 1631.

[26] M. Nogi, S. Iwamoto, A.N. Nakagaito, H. Yano, Adv. Mater. 21 (2009) 1595.

[27] M. Iftekhar Shams, S. Ifuku, M. Nogi, T. Oku, H. Yano, Appl. Phys. A 102 (2011) 325.

[28] S. Iwamoto, A.N. Nakagaito, H. Yano, M. Nogi, Appl. Phys. A 81 (2005) 1109.

[29] Y. Watanabe, S. Kitamura, K. Kawasaki, T. Kato, K. Uegaki, K. Ogura and K. Ishikawa, 
Biopolymers, 95 (2011) 833.

[30] J. Du, E. Tan, H.J. Kim, A. Zhang, R. Bhattacharya and K.J. Yarema, Carbohydr. Polym., 99 (2014) 483.

[31] W.-W. Hu and H.-N. Yu, Carbohydr. Polym., 95 (2013) 716.

[32] K.A. Rieger, N.P. Birch and J.D. Schiffman, J. Mater. Chem., B, 1 (2012) 4531.

[33] H. Kitano, H. Suzuki, K. Matsuura and K. Ohno, Langmuir, 26 (2010) 6767.

[34] H. Kitano, H. Suzuki, T. Kondo, K. Sasaki, S. Iwanaga, M. Nakamura, K. Ohno and Y. Saruwatari, Macromol. Biosci., 11 (2011) 557.

[35] T. Kamada, Y. Yamazawa, T. Nakaji-Hirabayashi, H. Kitano, Y. Usui, Y. Hiroi, T. Kishioka, Colloids Surf. B: Biointerfaces, 123 (2014) 878.

[36] H. Suzuki, L. Li, T. Nakaji-Hirabayashi, H. Kitano, K. Ohno, K. Matsuoka, Y. Saruwatari, Colloids Surf. B: Biointerfaces, 94 (2011) 107.

[37] K.J. Kubiak, M.C.T. Wilson, T.G. Mathia and Ph. Carval, Wear, 271 (2011) 523.

[38] H. Kitano, T. Kondo, T. Kamada, S. Iwanaga, M. Nakamura and K. Ohno, Colloids Surf. B: Biointerfaces, 88 (2011) 455 .

[39] T. Kondo, M. Gemmei-Ide, H. Kitano, K. Ohno, H. Noguchi and K. Uosaki, Colloids Surf. B: Biointerfaces, 91 (2012) 215.

[40] K. Nomura, T. Nakaji-Hirabayashi, M. Gemmei-Ide, H. Kitano, H. Noguchi and K. Uosaki, Colloids Surf. B: Biointerfaces, 123 (2014) 878.

[41] T. Kondo, K. Nomura, M. Murou, M. Gemmei-Ide, H. Kitano, K. Ohno, Y. Saruwatari, H. Noguchi and K. Uosaki, Colloids Surf. B: Biointerfaces, 100 (2012) 126.

[42] T. Kondo, K. Nomura, M. Gemmei-Ide, H. Kitano, H. Noguchi, K. Uosaki and Y. Saruwatari, Colloids Surf. B: Biointerfaces, 113 (2014) 361.

[43] L. Mi and S.Y. Jiang, Angew. Chem. Int. Ed. Engl., 53 (2014) 1746.

[44] H. Kitano, Polym. J., (2015) in press. 


\section{Figure captions}

Figure 1. Contact angle of the nanofiber (dark gray) and non-nanofiber (light gray) films composed of various contents of chitosan (Ch) and carboxymethyl cellulose (CMC).

Figure 2. Effect of chitosan $(\mathrm{Ch})$ nanofiber content on the $\zeta$-potential of nanofiber films. Data are represented as the mean \pm standard deviation $(\mathrm{N}=3)$.

Figure 3. Tensile strength $(\mathrm{MPa})$ of films constructed with various contents of chitosan $(\mathrm{Ch})$ and carboxymethyl cellulose (CMC) nanofibers (dark gray) or non-nanofibers (light gray). a) These data could not be obtained because of very low strength of the film. b) These samples could not be prepared. Data are shown as the mean \pm standard deviation for 6 independent samples. The symbols $*$ and $\ddagger$ indicate statistical significance (Tukey's HSD test, $* p<0.05$ and $\ddagger p<0.01$ ).

Figure 4. Number of fibroblasts (NIH 3T3) adhered to nanofiber films composed of various contents of chitosan (Ch) and carboxymethyl cellulose (CMC) nanofibers. Stained nuclei were observed by using a fluorescence microscope and counted on 5 different sites on a single sample. Data are shown as the mean \pm standard deviation for 4 independent samples.

Figure 5. Fluorescence micrographs of fibroblasts adhered to various nanofiber films; Chitosan (\%) $=0$ (a), 30 (b), 40 (c), 50 (d), 60 (e), 70 (f), and 100 (g). Scale bar $=500 \mu \mathrm{m}$. The cells were stained in green with calcein-AM to stain the cytosol of living cells.

Scheme 1. Chemical structure of (a) carboxymethyl cellulose sodium salt (CMC) and (b) chitosan (Ch). 


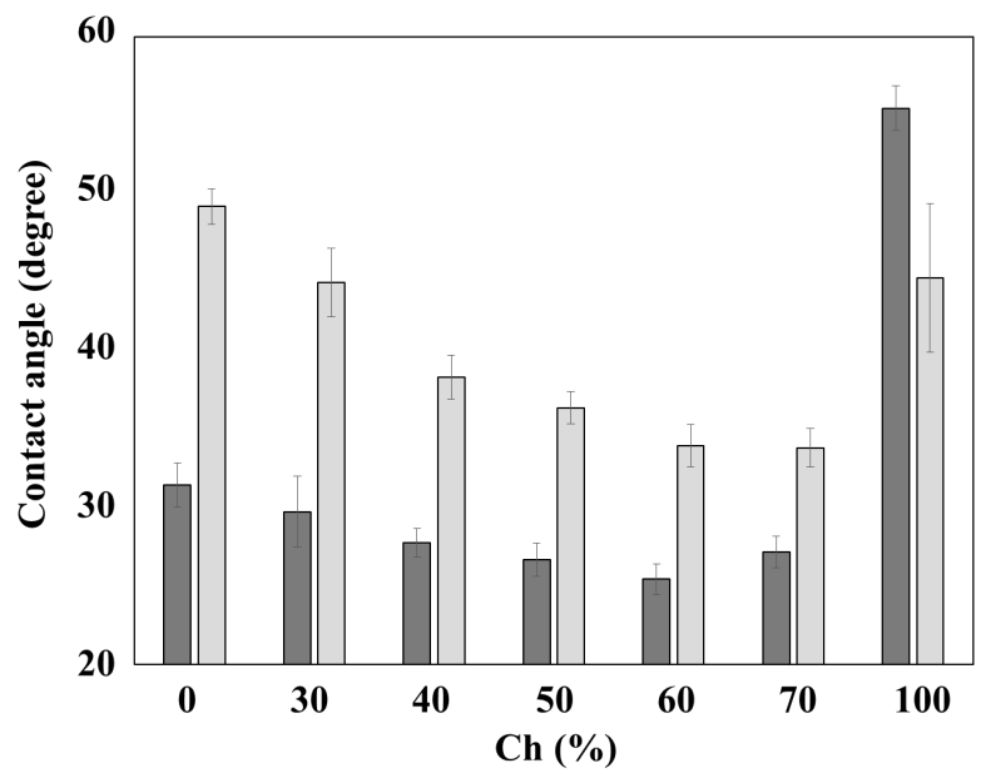

Figure 1. Contact angle of the nanofiber (dark gray) and non-nanofiber (light gray) films composed of various contents of chitosan (Ch) and carboxymethyl cellulose (CMC). 
Figure 2

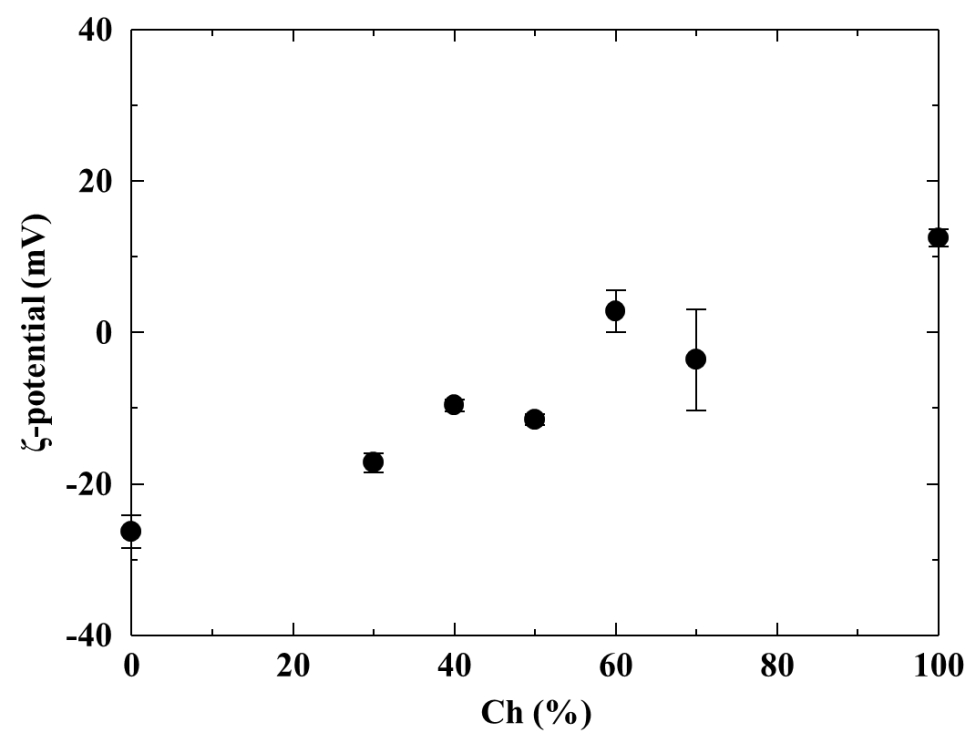

Figure 2. Effect of chitosan $(\mathrm{Ch})$ nanofiber content on the $\zeta$-potential of nanofiber films. Data are represented as the mean \pm standard deviation $(\mathrm{N}=3)$. 


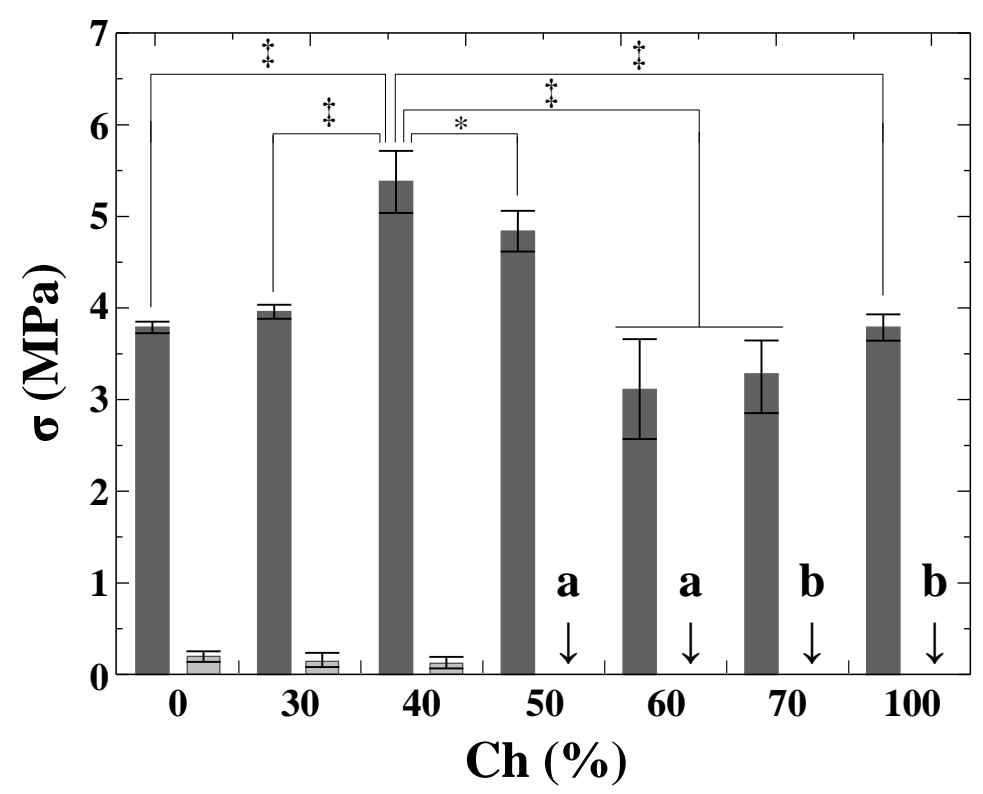

Figure 3. Tensile strength (MPa) of films constructed with various contents of chitosan (Ch) and carboxymethyl cellulose (CMC) nanofibers (dark gray) or non-nanofibers (light gray). a) These data could not be detected because of very low strength. b) These samples could not be prepared. Data are shown as the mean \pm standard deviation for 6 independent samples. The symbols $*$ and + indicate statistical significance (Tukey's HSD test, $* p<0.05$ and $\$ p<0.01$ ). 


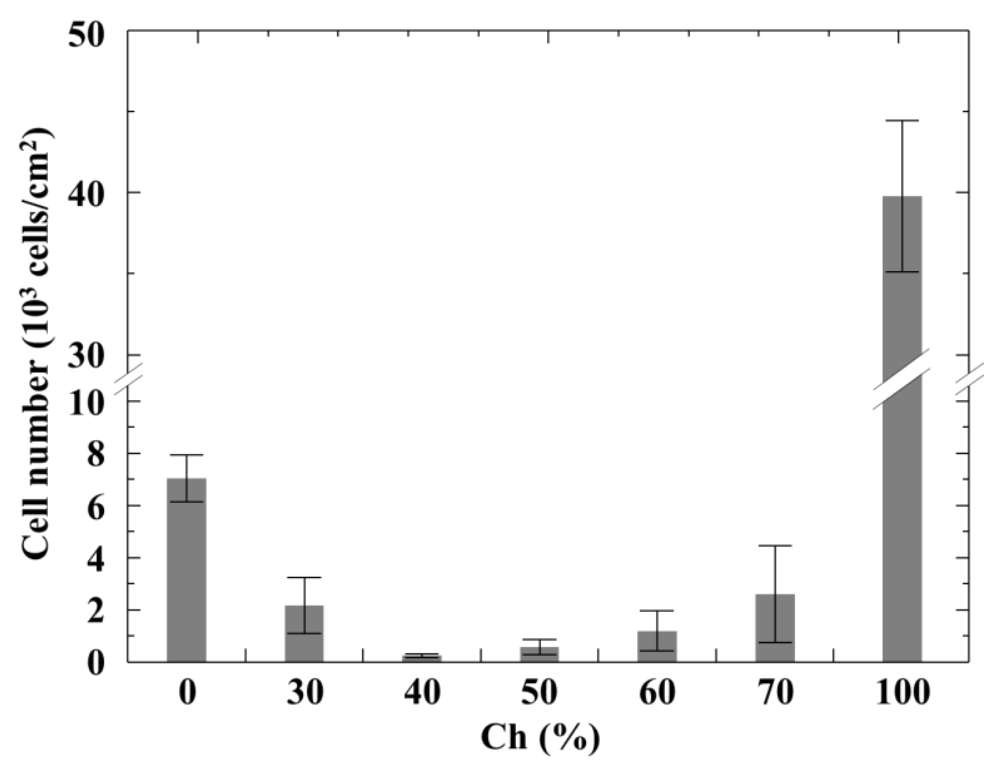

Figure 4. Number of fibroblasts (NIH 3T3) adhered to nanofiber films composed of various contents of chitosan (Ch) and carboxymethyl cellulose (CMC) nanofibers. Stained nuclei were observed by using a fluorescence microscope and counted on 5 different sites on a single sample. Data are shown as the mean \pm standard deviation for 4 independent samples. 

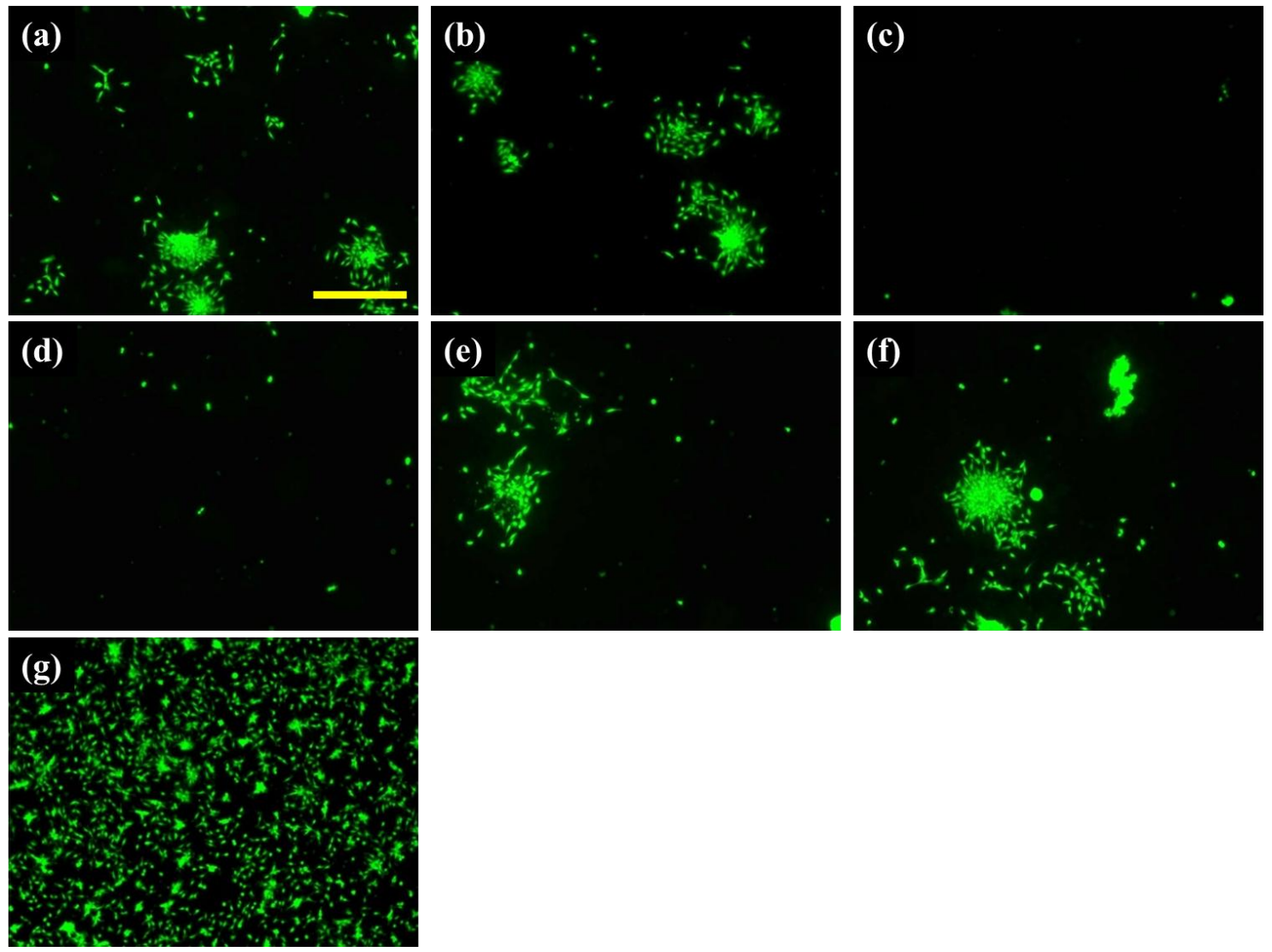

Figure 5. Fluorescence micrographs of fibroblasts adhered to various nanofiber films; Chitosan (\%) $=0$ (a), 30 (b), 40 (c), 50 (d), 60 (e), 70 (f), and 100 (g). Scale bar $=500 \mu \mathrm{m}$. The cells were stained in green with calcein-AM to stain the cytosol of living cells. 
2 Table 1. Characteristics of polymers examined.

\begin{tabular}{lll}
\hline Polymer & Degree of substitution $(\%)$ & Degree of deacetylation $(\%)$ \\
\hline NF CMC & 27.3 & - \\
Non-NF CMC & $47.7^{\mathrm{a}}$ & - \\
NF Ch & - & $92.0^{\mathrm{b}}$ \\
Non-NF Ch & - & $96.8^{\mathrm{c}}$ \\
\hline
\end{tabular}

${ }^{\mathrm{a}} 66.3-88.5 \%$ according to the manufacturer.

${ }^{\mathrm{b}} 92.1 \%$ according to the manufacturer.

${ }^{\mathrm{c}} 80.0 \mathrm{~mol} \%$ (minimum) according to the manufacturer. 
Table 2. Mechanical properties of the films composed of $\mathrm{Ch}$ and CMC nanofibers.

\begin{tabular}{lllll}
\hline Ch $(\%)$ & ${\text { Thickness }(\mathrm{mm})^{\mathrm{b}}}^{\mathrm{N}}$ & Width $(\mathrm{mm})$ & Elongation $(\%)$ & ${\text { Load }(\mathrm{gf})^{\mathrm{c}}}^{{ }^{2}}$ \\
\hline 0 & $0.117 \pm 0.024$ & 10 & 11.2 & $449 \pm 14.8$ \\
30 & $0.153 \pm 0.055$ & 5 & $-{ }^{\mathrm{a}}$ & $102 \pm 26.2$ \\
40 & $0.096 \pm 0.026$ & 10 & 18.3 & $515 \pm 64.6$ \\
50 & $0.086 \pm 0.024$ & 10 & 14.2 & $431 \pm 39.4$ \\
60 & $0.114 \pm 0.041$ & 10 & 15.3 & $356 \pm 24.6$ \\
70 & $0.160 \pm 0.058$ & 5 & $-{ }^{\mathrm{a}}$ & $166 \pm 28.4$ \\
100 & $0.087 \pm 0.029$ & 10 & 11.6 & $333 \pm 25.2$ \\
\hline
\end{tabular}

${ }^{a}$ Not detected. ${ }^{b}$ The thickness of nanofiber films was measured at 6 different positions on a single sample and averaged from 3 different samples. ${ }^{\mathrm{c}}$ The data are shown as the mean \pm standard deviation for 3 independent samples. 


\section{Graphical Abstract}

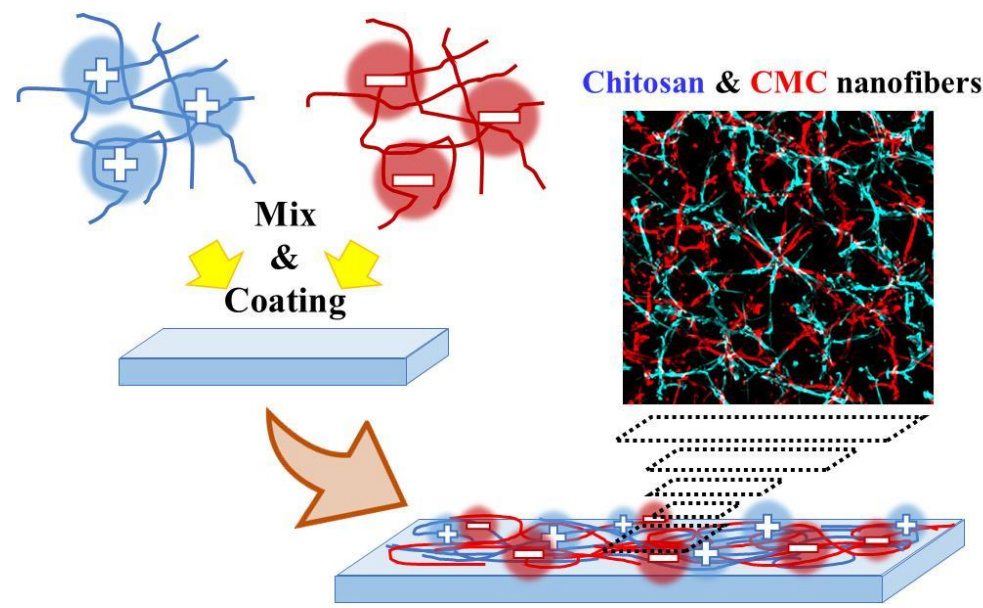

Schematic illustration of film of polyion complex with Ch- and CMC- nanofibers 